\title{
Targeting melanoma with front-line therapy does not abrogate Nodal-expressing tumor cells
}

\author{
Mary JC Hendrix ${ }^{1,2,3}$, Irawati Kandela ${ }^{4}$, Andrew P Mazar ${ }^{4}$, Elisabeth A Seftor ${ }^{2,3}$, Richard EB Seftor ${ }^{2,3}$, \\ Naira V Margaryan²,3, Luigi Strizzi ${ }^{2,5}$, George F Murphy ${ }^{6}$, Georgina V Long ${ }^{7}$ and Richard A Scolyer 7,8
}

Metastatic melanoma is a highly aggressive skin cancer with a poor prognosis. It is the leading cause of skin cancer deaths with a median overall survival for advanced-stage metastatic disease of $<6$ months. Despite advances in the field with conventional and targeted therapies, the heterogeneity of melanoma poses the greatest ongoing challenge, ultimately leading to relapse and progression to a more drug-resistant tumor in most patients. Particularly noteworthy are recent findings, indicating that these therapies exert selective pressure on tumors resulting in the activation of pathways associated with cancer stem cells that are unresponsive to current therapy. Our previous studies have shown how Nodal, an embryonic morphogen of the transforming growth factor-beta superfamily, is one of these critical factors that is reactivated in aggressive melanoma and resistant to conventional chemotherapy, such as dacarbazine. In the current study, we sought to determine whether BRAF inhibitor (BRAFi) therapy targeted Nodal-expressing tumor cells in uniquely matched unresectable stage III and IV melanoma patient samples before and after therapy that preceded their eventual death due to disease. The results demonstrate that BRAFi treatment failed to affect Nodal levels in melanoma tissues. Accompanying experiments in soft agar and in nude mice showed the advantage of using combinatorial treatment with BRAFi plus anti-Nodal monoclonal antibody to suppress tumor growth and metastasis. These data provide a promising new approach using front-line therapy combined with targeting a cancer stem cell-associated molecule-producing a more efficacious response than monotherapy.

Laboratory Investigation (2017) 97, 176-186; doi:10.1038/labinvest.2016.107; published online 24 October 2016

Melanoma remains the most aggressive and deadly form of skin cancer with a median overall survival for advanced-stage metastatic disease of $<6$ months. ${ }^{1}$ Traditional treatment approaches for patients with advanced-stage disease have included conventional chemotherapy such as dacarbazine (DTIC), with questionable survival benefit to patients. ${ }^{2,3}$ More recently, FDA-approved agents have included ipilimumab, a mAb that targets a regulatory checkpoint, CTLA-4, in $\mathrm{T}$ cells, that improves overall survival. ${ }^{4}$ In addition, the identification of mutationally activated BRAF in melanoma and many other cancers has led to the development of BRAFi's, such as vemurafenib and dabrafenib, that have resulted in high response rates in patients whose tumor harbor such mutations. ${ }^{5,6}$ Trametinib, which is approved for patients whose tumors express the BRAF V600E or V600K gene mutations, ${ }^{7}$ inhibits the mitogen-activated, extracellular signal-regulated kinase inhibitor (MEK) downstream of BRAF and is usually administered in combination with a BRAFi. Other recent therapeutic breakthroughs include targeting of the programmed death 1 pathway. ${ }^{8}$ Although these therapies have improved the prospects of melanoma patients, some patients do not respond and others relapse despite initial responses. Therefore, an urgent clinical need remains that justifies our continuing quest to identify more efficient therapeutic approaches to achieve improved progression-free and overall survival.

In aggressive tumors, one of the most difficult challenges to address is cellular heterogeneity. As such, when a

\footnotetext{
'Department of Biology, Shepherd University, Shepherdstown, W, USA; ${ }^{2}$ Program in Cancer Biology and Epigenomics, Stanley Manne Children's Research Institute at Ann and Robert H. Lurie Children's Hospital of Chicago, Northwestern University Feinberg School of Medicine, Chicago, IL, USA; ${ }^{3}$ Robert C. Byrd Health Sciences Center, West Virginia University Cancer Institute, West Virginia University, Morgantown, WV, USA; ${ }^{4}$ Department of Pharmacology, Feinberg School of Medicine, Northwestern University, Evanston, IL, USA; ${ }^{5}$ Department of Pathology, Midwestern University, Downers Grove, IL, USA; ${ }^{6}$ Department of Pathology, Harvard Medical School, Brigham and Women's Hospital, Boston, MA, USA; ${ }^{7}$ Melanoma Institute Australia and Sydney Medical School, The University of Sydney, Sydney, NSW, Australia and ${ }^{8}$ Department of Tissue Pathology and Diagnostic Oncology, Royal Prince Alfred Hospital, Sydney, NSW, Australia

Correspondence: MJC Hendrix, PhD, Department of Biology, Shepherd University, PO Box 5000, 301N King Street, Shepherdstown, WW 25443, USA or Robert C. Byrd Health Sciences Center, West Virginia University Cancer Institute, West Virginia University, Morgantown, WV 26506, USA.

E-mail: mhendrix@shepherd.edu
}

Received 15 July 2016; revised 24 August 2016; accepted 6 September 2016 
heterogeneous tumor responds to monotherapy, the outcome is often an initial response but is often accompanied by expansion of tumor cells with stem cell properties, ultimately leading to relapse and progression of a more drug-resistant tumor. ${ }^{9}$ There is supportive evidence indicating that the selective pressure exerted by targeted agents can lead to the activation of or selection of cancer cells with alternative signaling events, resulting in disease progression that is unresponsive to therapy. ${ }^{10,11}$ Efforts to address this challenge are particularly noteworthy in the management of melanoma where combinatorial regimens and sequencing of therapies will likely lead to further improvements in outcomes. ${ }^{12}$ However, the identification of novel targetable molecules that contribute to the cancer stem cell, drug-resistant phenotype are highly desired for therapeutic development.

Our studies have demonstrated how Nodal, an embryonic morphogen of the transforming growth factor-beta (TGF $\beta$ ) superfamily, can have a critical role in aggressive human cancer, specifically with regard to tumor growth, metastasis, the cancer stem cell phenotype, and resistance to conventional therapy, such as DTIC and vemurafenib., ${ }^{9}, 13-18$ Our findings, as well as those by others, have shown significant levels of Nodal expression in a variety of virulent neoplasms, including melanoma, breast, prostate, ovary, and ovarian cancers in association with advanced disease. ${ }^{19}$ Because Nodal expression is not typically observed in most normal adult tissues, it has the potential as a promising new targetable molecule during the late stages of cancer progression. In the current study, we addressed a basic question related to the potential targeting of Nodal-expressing tumor cells in uniquely matched AJCC (American Joint Committee on Cancer) unresectable stage III and IV melanoma patient samples before and after BRAFi therapy that preceded their eventual death due to disease. The results indicate that BRAFi treatment failed to affect Nodal levels in melanoma tissues. Follow-up experimental studies in soft agar and in nude mice demonstrated the benefit of using combinatorial treatment with BRAFi plus anti-Nodal monoclonal antibody (mAb) to suppress tumor growth and metastasis, thus providing a novel approach using front-line therapy combined with targeting a cancer stem cell-associated molecule.

\section{MATERIALS AND METHODS}

\section{Clinical Melanoma Samples}

Multiple tumor samples were collected from 17 patients with BRAF mutant unresectable AJCC stage III or stage IV melanoma during the course of their treatment with either a BRAFi alone (vemurafenib or dabrafenib, BRAF mono, $N=13$ ) or the combination of BRAF and MEK inhibitor (dabrafenib and trametinib, BRAFi combo) as part of clinical trials accrued between 2009 and 2013 (Table 1). The BRAF mutation status was determined as previously described. ${ }^{20,21}$ Biopsied tumor specimens were collected from consenting patients before MAPK inhibitor treatment (PRE) and at disease progression (POST), with Human Ethics Review
Table 1 Number of patients and treatment received

A

\begin{tabular}{lcccc}
\hline Treatment & Dabrafenib (d) & Vemurafenib (v) & $\begin{array}{c}\text { Dabrafenib } \\
\text { +trametinib }(\mathrm{d}+\mathrm{t})\end{array}$ & Total \\
\hline $\mathrm{N}$ & 12 & 1 & 4 & 17
\end{tabular}

B

\begin{tabular}{|c|c|c|c|}
\hline Case & BRAF mutation analysis & Sample & Nodal IHC index score \\
\hline \multirow[t]{2}{*}{$1(\mathrm{~d})$} & BRAF V600E & Pre-Tx & 9 \\
\hline & & Post-Tx & 9 \\
\hline \multirow[t]{2}{*}{$2(d)$} & BRAF V600K & Pre-Tx & 9 \\
\hline & & Post-Tx & 9 \\
\hline \multirow[t]{2}{*}{$3(d)$} & BRAF V600E & Pre-Tx & 4 \\
\hline & & Post-Tx & 1 \\
\hline \multirow[t]{2}{*}{$4(d)$} & BRAF V600E & Pre-Tx & 4 \\
\hline & & Post-Tx & 4 \\
\hline \multirow[t]{2}{*}{$5(v)$} & BRAF V600E & Pre-Tx & 3 \\
\hline & & Post-Tx & 6 \\
\hline \multirow[t]{2}{*}{$6(d+t)$} & BRAF V600K & Pre-Tx & 9 \\
\hline & & Post-Tx & 9 \\
\hline \multirow[t]{2}{*}{$7(d+t)$} & BRAF V600E & Pre-Tx & 9 \\
\hline & & Post-Tx & 4 \\
\hline \multirow[t]{2}{*}{$8(d)$} & BRAF V600E & Pre-Tx & 9 \\
\hline & & Post-Tx & 1 \\
\hline \multirow[t]{2}{*}{9 (d) } & BRAF V600K & Pre-Tx & 4 \\
\hline & & Post-Tx & 4 \\
\hline \multirow[t]{2}{*}{$10(d)$} & BRAF V600E & Pre-Tx & 6 \\
\hline & & Post-Tx & 4 \\
\hline \multirow[t]{2}{*}{$11(d)$} & BRAF V600K & Pre-Tx & 6 \\
\hline & & Post-Tx & 9 \\
\hline \multirow[t]{2}{*}{$12(d+t)$} & BRAF V600K & Pre-Tx & 6 \\
\hline & & Post-Tx & 4 \\
\hline \multirow[t]{2}{*}{$13(d)$} & BRAF V600E & Pre-Tx & 2 \\
\hline & & Post-Tx & 9 \\
\hline \multirow[t]{2}{*}{$14(\mathrm{~d}+\mathrm{t})$} & BRAF V600E & Pre-Tx & 1 \\
\hline & & Post-Tx & 4 \\
\hline \multirow[t]{2}{*}{$15(d)$} & BRAF V600E & Pre-Tx & 1 \\
\hline & & Post-Tx & 1 \\
\hline \multirow[t]{2}{*}{$16(d)$} & BRAF V600E & Pre-Tx & 1 \\
\hline & & Post-Tx & 1 \\
\hline \multirow[t]{2}{*}{17 (d) } & BRAF V600E & Pre-Tx & 1 \\
\hline & & Post-Tx & 6 \\
\hline
\end{tabular}


Committee approval as part of the Treat Excise Analyze for Melanoma (TEAM) study at Melanoma Institute Australia. ${ }^{22,23}$ Clinical and follow-up details were collated and analyzed on all patients. All slides were devoid of any patient identifier and histological and immunohistochemistry analysis performed in compliance with IRB approval (Lurie Children's approval number 2006-12921).

\section{Source of Cells}

The highly metastatic A375SM-L1 human melanoma cell line, which harbors the active BRAF (V600E) mutation, ${ }^{24}$ was a kind gift from Dr Menashe Bar-Eli, University of Texas, MD Anderson Cancer Center. The cell line was authenticated by short tandem repeat genotyping at the Ann and Robert $\mathrm{H}$. Lurie Children's Hospital of Chicago molecular diagnostics core, routinely tested for mycoplasma contamination with a PCR ELISA kit (Roche Applied Science, Indianapolis, IN, USA), and maintained as previously described. ${ }^{19}$

\section{Animal Regulation Compliance}

The Institutional Animal Care and Use Committee (IACUC) reviews and approves protocols for Northwestern University's program for the humane care and use of animals and inspects the animal facilities and investigator laboratories. Evaluation of WS65 antibody alone or in combination with dabrafenib in the A375SM-L1 metastasis xenograft model was performed as described below in compliance with Animal Welfare and Northwestern's IACUC regulations under approved IACUC protocol IS00000556 'In Vivo Assessment of Efficacy for Novel Compounds in the Treatment of Cancer: Orthotopic tumor model.'

\section{A375SM-L1 Tumor Xenografts}

A375SM-L1 cells were transfected with Luc2, maintained in RPMI 1640 with $10 \%$ FBS and $0.1 \%$ gentamycin sulfate before inoculation. The viability of the cells before inoculation was assessed and determined to be $>97 \%$. Female athymic nude mice CrTac:NCr-Foxn1 ${ }^{\text {nu }}$ (Taconic Bioscience, Germantown, NY, USA) weighing 17-20g were inoculated intravenously with $2.5 \times 10^{5}$ A375SM-L1 cells via tail vein injection. Mice were monitored using IVIS (Perkin-Elmer, Waltham, MA, USA) imaging for tumor growth in the lungs, as previously described. ${ }^{25}$ IVIS has been shown to faithfully correlate with tumor growth in vivo. ${ }^{26}$ Once the bioluminescence signal was detected, the mice were randomized into five different groups and treatment initiated (Table 2). Treatment was maintained, as per the schedule described in Table 2, for the duration of the study. Mice were checked twice daily post injection for mortality, abnormalities, and sign of pain or distress. Each cage contained three to five female mice, and offered chow (Certified Rodent Diet \#7912, Harlan, Indianapolis, IN, USA) and water ad libitum unless otherwise specified. Environmental controls for the animal room were maintained between 68 and $75^{\circ} \mathrm{F}$, a relative humidity of $30-70 \%$ and a minimum of 15 room air changes per hour 
and 12-h light/12-h dark cycle. The light/dark cycle was interrupted for study-related activities.

The effects of study drug on tumor growth were evaluated during the in-life phase by IVIS imaging (radiance) ${ }^{26}$ and by lung weights after euthanasia and excision from each mouse. Mice were killed at day 21 and lungs were removed, weighed, and photographed. Excised lungs were cut into two equal segments with half fixed in formalin for immunohistochemistry (IHC) and half flash-frozen for molecular analysis. Lung weights were recorded for each group and differences analyzed using one-way ANOVA analysis (mean \pm s.e.m., GraphPad Software, Inc., La Jolla, CA, USA).

\section{Anchorage-Independent Colony Formation Assay (Clonogenic Assay)}

Assays were prepared in triplicate as previously described. ${ }^{19}$ A375SM-L1 cells were either untreated (control), or treated with $1 \mu \mathrm{g} / \mathrm{ml}$ of mouse IgG (Jackson ImmunoResearch, West Grove, PA, USA; IgG control), or DMSO alone (DMSO control), or $1 \mu \mathrm{g} / \mathrm{ml}$ of a monoclonal anti-Nodal antibody (WS65, Santa Cruz Biotechnology, Dallas, TX, USA), or $1 \mathrm{nM}$ dabrafenib (LC Laboratories, Woburn, MA, USA), or a combination of both $1 \mathrm{nM}$ dabrafenib and $1 \mu \mathrm{g} / \mathrm{ml}$ of WS65 for $72 \mathrm{~h}$, then 5000 cells were suspended in $0.35 \%$ agarose in RPMI 1640 (Life Technologies, Grand Island, NY, USA) plus $10 \%$ serum, and then overlaid onto a solidified layer of $0.5 \%$ agar in RPMI 1640 plus $10 \%$ serum in six-well dishes. After 3 weeks, cell clusters ( $\geq 50$ cells per cluster) were counted in triplicate wells and the averages from separate experiments presented as a percentage (mean \pm s.e.m.) of the no treatment control. Statistical analyses were performed using the Microsoft Excel spreadsheet statistical package and Student's $T$-test used to determine statistical significance using $P \leq 0.05$ as biologically significant.

\section{Western Blot Analysis}

A375SM-L1 cells were either untreated, or treated with an IgG control, dabrafenib, anti-Nodal mAb (WS65), or both dabrafenib and WS65 mAb for $72 \mathrm{~h}$, then whole-cell lysates prepared in RIPA buffer containing a protease inhibitor cocktail (Complete Mini, Roche Diagnostics) with sonication (Supplementary Figure 2). After centrifugation at 13000 r.p. $\mathrm{m}$. for $30 \mathrm{~min}$, the protein concentration of the supernatants was determined using a BCA assay (Thermo Scientific, Rockford, IL, USA) and $40 \mu \mathrm{g}$ of protein loaded per well of a $4-12 \%$ NuPage electrophoresis gel (Life Technologies). After electrophoresis, the proteins were electroblotted onto an Immobilon-P membrane (Millipore, Billerica, MA, USA). Nodal protein was detected in its more stable ProNodal form using a rabbit monoclonal anti-Nodal antibody (Abcam, Clone EP2058Y, Cambridge, MA, USA) followed by an antirabbit plus horseradish peroxidase (HRP) secondary antibody (GE Healthcare, Pittsburgh, PA, USA, NA934V) and enhanced chemiluminescence (ECL; GE Healthcare). The blot was then stripped and $\beta$-actin protein detected using a mouse anti- $\beta$-actin mAb (Millipore, Temecula, CA, USA, MAB1501), an anti-mouse plus HRP secondary antibody (GE Healthcare) and ECL as a control for the amount of protein loaded per lane. The exposed films were digitized using a ChemiDoc XRS imager (Bio-Rad Laboratories, Hercules, CA, USA) and the relative amount of protein determined against the untreated control and IgG-treated cells normalized to a value of 1.0 using the imager's Quantity One software package (Bio-Rad Laboratories) corrected for loading against the $\beta$-actin protein control.

\section{Immunohistochemistry}

Four micron-thick, formalin-fixed, paraffin-embedded tissue sections on de-identified slides were used for IHC analysis of Nodal expression. IHC staining was performed using a Dako Plus autostainer (Dako, Inc, Carpinteria, CA, USA) as previously described. ${ }^{19}$ In brief, following antigen retrieval and blocking steps, sections were incubated in a goat antihuman Nodal antibody (LS-B3955; LifeSpan Biosciences, Seattle, WA, USA, 1:150) for mouse xenografts and the mouse monoclonal anti-Nodal antibody (ab55676, Abcam, 1:200) for human tissue sections for $60 \mathrm{~min}$, followed by appropriate biotinylated secondary antibody (Biocare Medical, Concord, CA, USA), and then streptavidin-horseradish peroxidase (Thermo Scientific Lab Vision, Fremont, CA, USA). Immunostaining was detected using either 3,3'-diaminobenzidine (Thermo Scientific Lab Vision) or 3-amino-9-ethylcarbazole (Biocare Medical) peroxidase chromogen substrates. Sections were counterstained with hematoxylin (Biocare Medical). As a negative control, adjacent serial sections were incubated with species appropriate irrelevant IgG (Jackson ImmunoResearch Labs) at the same concentration as primary antibodies. Sections were reviewed and scored as previously described. ${ }^{27}$

\section{RESULTS}

Nodal Expression is Not Targeted by BRAFi in Melanoma Patient Tumors

A comparative analysis of Nodal expression by IHC was performed (in a blinded manner) on matched stage 4 melanoma tissue samples from patients before (PRE) and on disease progression (POST) after treatment with BRAFi's used as a monotherapy (dabrafenib, $N=12$; vemurafenib, $N=1$ ) or in a combinatorial manner (dabrafenib plus trametinib-a MEK inhibitor, $N=4$, Table 1). Regardless of patient gender, age, or type of mutation at diagnosis, there was no significant difference in Nodal-expressing tumor cells before, or on disease progression after BRAFi monotherapy or combination therapy, as shown in Figure 1 and Supplementary Figure 1. The individual Nodal IHC scores reported in Table 1 are summarized in histogram form comparing mean Nodal IHC scores of matched excision biopsies taken from the same melanoma patient pre-BRAFi treatment and post-BRAFi treatment (Nodal score pre$\mathrm{BRAFi}=4.94 \pm 3.2 v s$ Nodal score post $\mathrm{BRAFi}=5.29 \pm 2.9$, 
$N=17, P=0.74)$. Representative Nodal IHC staining at the microscopic level is shown in Supplementary Figure 1 and demonstrates the intensity and localization of Nodal expression. Together, these data indicate that the front-line therapy of dabrafenib or vemurafenib, or dabrafenib plus the MEK inhibitor trametinib, does not significantly influence Nodal-

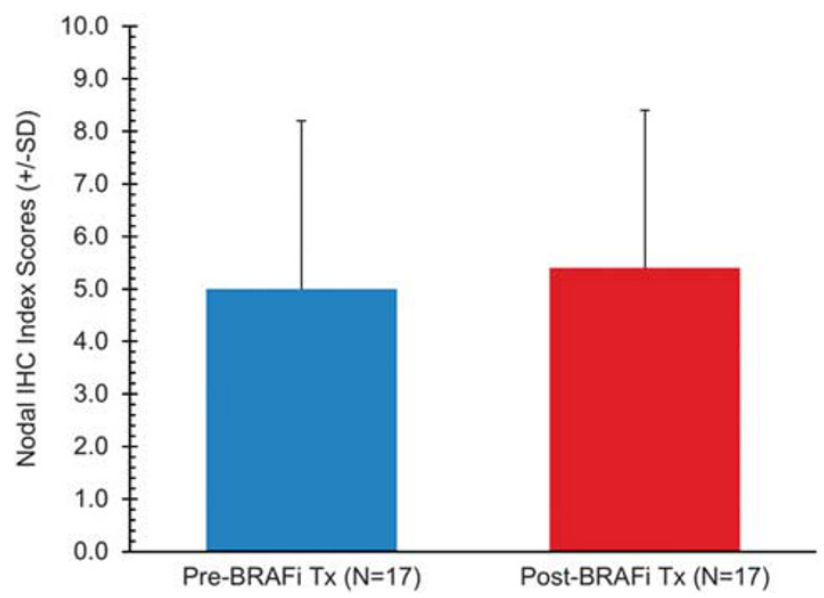

Figure 1 Nodal expression is not targeted by BRAFi in melanoma tumors. The histogram represents comparison of Nodal protein expression in matched melanoma tissue samples from patients before (PRE) and after treatment on disease progression (POST) with BRAF inhibitors (BRAFi), and shows no significant difference in Nodal expression in melanoma tissues before and after treatment $(P=0.74)$. expressing melanoma tumor cells in patients with advancedstage disease.

\section{Anchorage-Independent Colony Formation (Clonogenic) Potential of Melanoma Cells is Suppressed by Combination Treatment with BRAFi Plus Anti-Nodal mAb} Based on the Nodal findings in melanoma patients, we embarked on an experimental approach to test the hypothesis that targeting Nodal together with a front-line therapy would reduce tumor growth properties and ultimately metastasis. In these experiments, we chose to use the highly metastatic A375SM-L1 human melanoma cell line, which harbors the active BRAF (V600E) mutation, and treated them with dabrafenib alone or with a combination of dabrafenib plus an anti-Nodal mAb WS65. The findings demonstrate that, although the anchorage-independent colony formation growth potential of A375SM-L1 cells in soft agar is significantly reduced in tumor colony size and number after treatment with dabrafenib or WS65 mAb, the most marked inhibition results occurred with the combination treatment of dabrafenib plus WS65 mAb, compared with their respective controls (Figure 2a). Particularly important was the significant effect elicited by the very low concentration of dabrafenib and WS65 mAb, where western blot analysis indicated Nodal expression to be reduced by $37 \%$ with dabrafenib only, by 44\% with WS65 mAb, and by 76\% with dabrafenib plus WS65 mAb (Supplementary Figure 2). Relative colony forming ability was measured as a percentage a

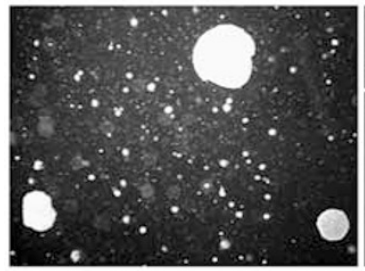

No Treatment Control

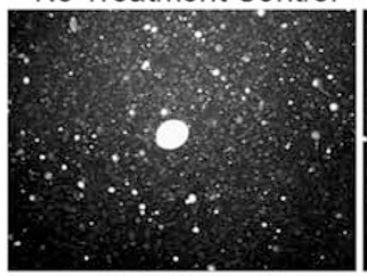

$1 \mathrm{nM}$ Dabrafenib

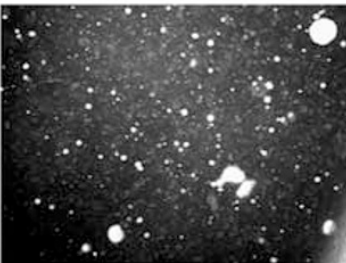

IgG Control

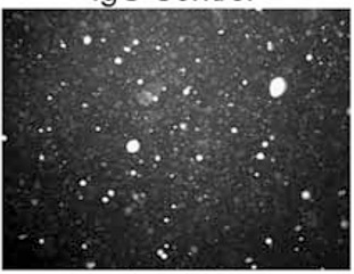

$1 \mu \mathrm{g} / \mathrm{ml}$ WS65

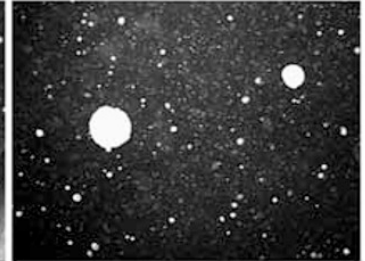

DMSO Control

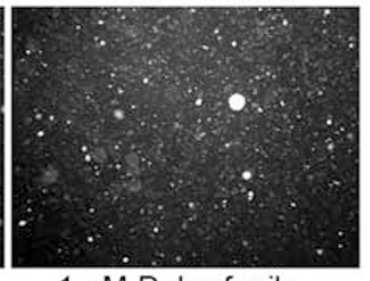

$1 \mathrm{nM}$ Dabrafenib

$+1 \mu \mathrm{g} / \mathrm{ml}$ WS65 b

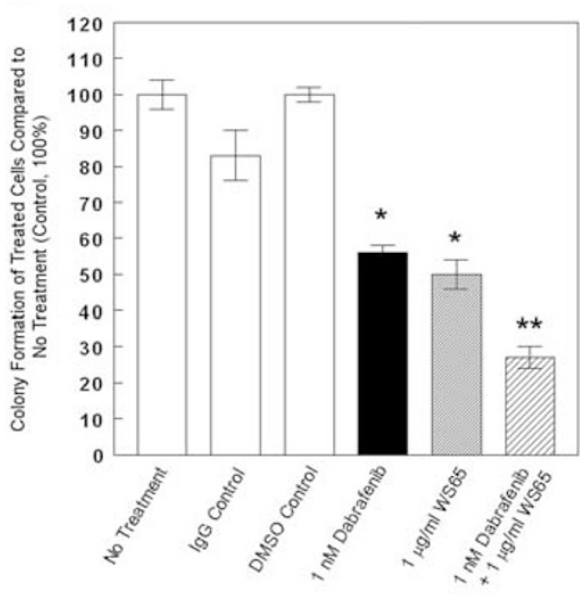

Figure 2 Anchorage-independent colony formation (clonogenic) potential of melanoma cells is most inhibited by combination treatment with BRAFi plus anti-Nodal mAb. Changes in the anchorage-independent growth (clonogenicity) of A375SM-L1 cells in response to treatment with an anti-Nodal mAb (WS65), dabrafenib or a combination of both 3 weeks after treatment are evaluated. (a) Representative images of the A375SM-L1 cell clusters formed in soft agar 3 weeks after removal from treatment with WS65 mAb, dabrafenib or a combination compared with no treatment (control) or treatment with IgG (IgG control) or DMSO alone (DMSO control). (b) Relative colony formation of A375SM-L1 cells cultured on soft agar ( $\geq 50$ cells per cluster, shown in a) following 72-h treatment with $1 \mu \mathrm{g} / \mathrm{ml}$ WS65 mAb, $1 \mathrm{nM}$ dabrafenib, or a combination of both $1 \mathrm{nM}$ dabrafenib and $1 \mu \mathrm{g} / \mathrm{ml}$ of WS65 mAb compared with untreated control cells were determined as a percentage (mean \pm s.e.m.) of untreated control cells (* $P \leq 0.05$ ). The significance in colony formation between either the $1 \mu \mathrm{g} / \mathrm{ml}$ WS65 mAb or $1 \mathrm{nM}$ dabrafenib treatments and the combination of both is also significantly different $\left({ }^{* *} P \leq 0.05\right)$. 
of tumor clusters formed in soft agar 3 weeks after removal from a 72 -h treatment with $1 \mathrm{nM}$ dabrafenib, $1 \mu \mathrm{g} / \mathrm{ml}$ WS65 $\mathrm{mAb}$, or combined $1 \mathrm{mM}$ dabrafenib plus $1 \mu \mathrm{g} / \mathrm{ml}$ WS65 $\mathrm{mAb}$, compared with controls (Figure 2b). Treatment with $1 \mathrm{mM}$ dabrafenib resulted in $56 \%$ tumor colony growth compared with DMSO control, whereas melanoma cells treated with $1 \mu \mathrm{g} / \mathrm{ml}$ WS65 mAb displayed 50\% tumor colony growth compared with the IgG control. Most noteworthy, the melanoma cells receiving combinatorial treatment with $1 \mathrm{mM}$ dabrafenib plus $1 \mu \mathrm{g} / \mathrm{ml}$ WS65 mAb achieved only $27 \%$ tumor colony growth compared with the control. Collectively, these data indicate that the most significant reduction in tumorigenic potential occurs when melanoma is treated with a frontline BRAFi plus anti-Nodal mAb.

\section{Combinatorial Treatment with Anti-Nodal mAb and Dabrafenib Suppresses Lung Metastases}

Table 2 describes the dose, schedule, and route of administration for the various treatment groups evaluated in this study. In brief, WS65 anti-Nodal $\mathrm{mAb}(2.5 \mathrm{mg} / \mathrm{kg} \mathrm{IP})$ was

\section{Group 1: Non-specific IgG}

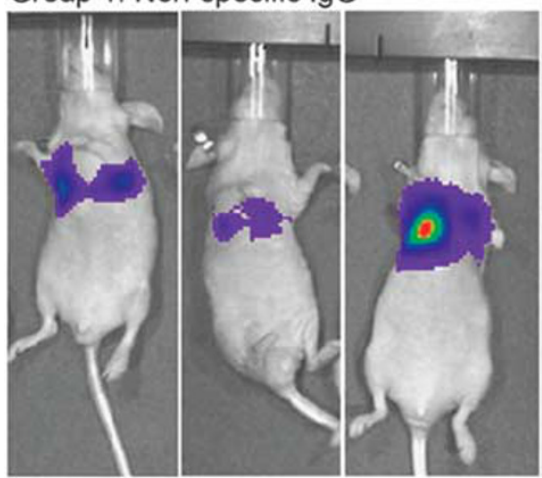

Group 3: WS65 anti-Nodal Ab (mAb)
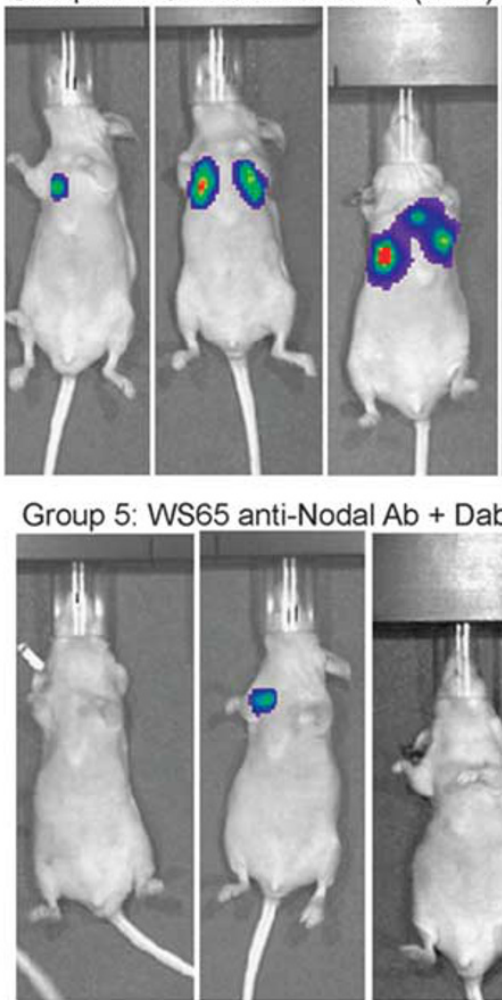
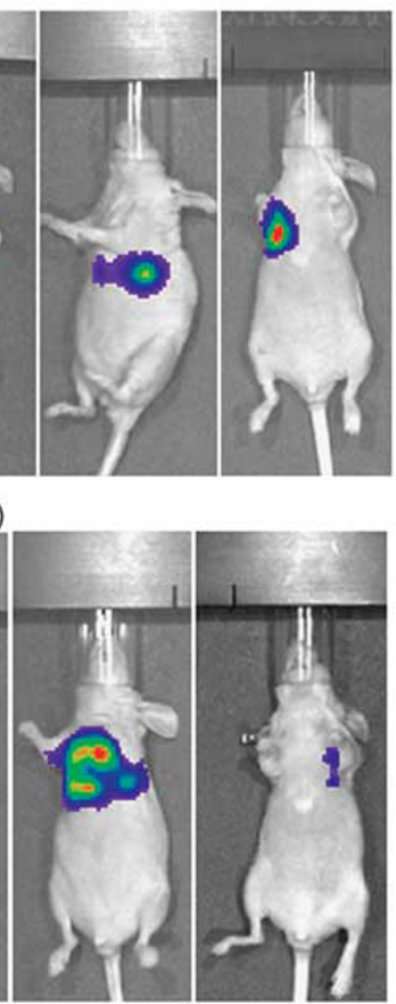

$A b+$ Dabrafenib
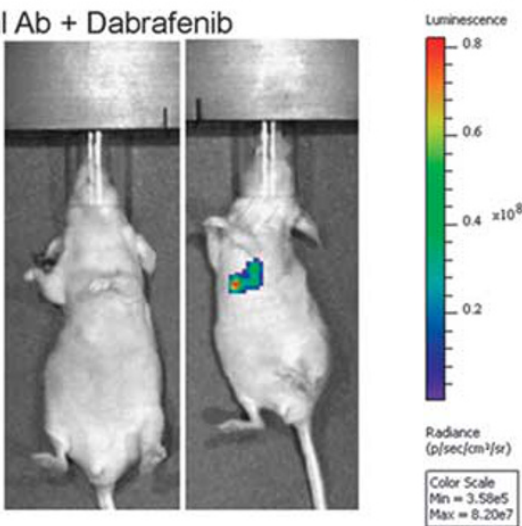

\section{Group 2: Dabrafenib vehicle}

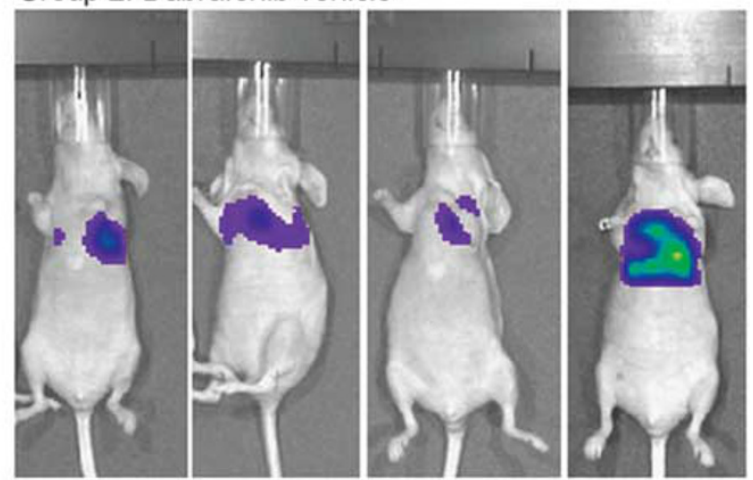

Group 4: Dabrafenib

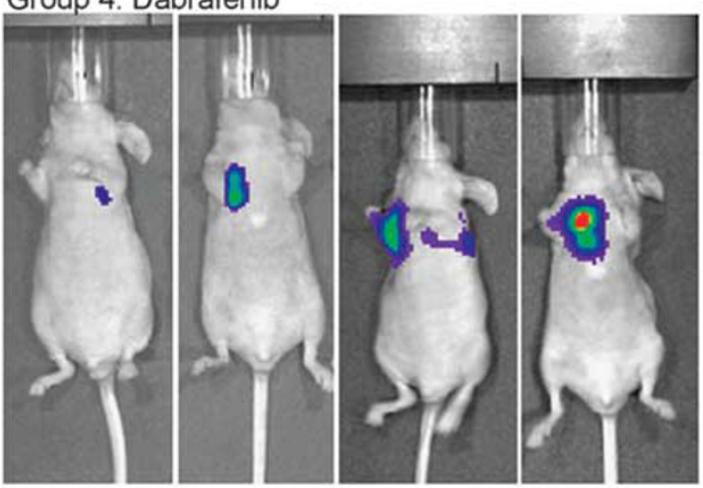

Figure 3 Progression of A375SM-L1 lung metastasis is monitored by IVIS imaging during in-life phase. At the end of the study (21 days), mice were killed using isoflurane, placed in a supine position, and images acquired in auto mode in the IVIS system (Perkin-Elmer). Images were processed using Living Image software (version 4.5) and data were recorded as the average radiance $\left(\mathrm{p} / \mathrm{s} / \mathrm{cm}^{2} / \mathrm{sr}\right.$ ) as previously described. ${ }^{22}$ 
administered via IP injection once per week and dabrafenib ( $3 \mathrm{mg} / \mathrm{kg}$ PO) was administered on Monday, Wednesday, and Friday. Non-specific antibody IgG1 $(2.5 \mathrm{mg} / \mathrm{kg})$ was given IP as a control for WS65 mAb-treated mice and dabrafenib vehicle was provided via oral gavage at the same volume as dabrafenib-treated mice ( $200 \mu \mathrm{l} / 20 \mathrm{~g}$ mouse). All treatments were administered for the full 21 days of the study before euthanasia. Animals were weighed before dosing. Bioluminescence signals from the lungs were obtained weekly using IVIS and mice were checked for clinical signs daily to assess treatment tolerability/toxicity and the anti-tumor effects of the therapy regimens. Treatment effect was monitored during the in-life phase using the bioluminescence signal from IVIS (Figure 3) and lung weight was used to quantitate the antitumor activity of each treatment at the end of the study (Figure 4a). No adverse effects were observed in terms of increased mortality or clinical signs during the course of the study and no overt toxicity was observed by body weight assessments (Figure 4b) in any of the treatment groups. All animals survived until the end of the study. Treatment with WS65 mAb (group 3) or dabrafenib (group 4) monotherapy decreased lung weights by $\sim 30 \%$ (Figure $4 \mathrm{a}$ ). However, the combination of WS65 mAb and dabrafenib (group 5) decreased lung weights by $\sim 60 \%$, and gross evaluation of lungs showed little evidence of macroscopic tumor growth. Histological evaluation of H\&E-stained sections and Nodal IHC from the WS65 mAb plus dabrafenib-treated lungs demonstrates almost completely normal lung histology with only occasional evidence of micrometastatic lesions (Figure 5). Lungs from mice treated with either WS65 mAb or dabrafenib monotherapy show large metastatic lesions, and control-treated groups show near complete replacement of normal lung tissue with tumor in some sections (Figure 5). Despite the large amount of tumor burden in the controltreated mice, overt evidence of distress was prevented by terminating the studies after 21 days consistent with the previous studies in experimental metastasis models, demonstrating that mice can tolerate large lung tumor burdens before showing overt distress. ${ }^{28}$ Collectively, the in vivo experimental findings demonstrate a noteworthy suppression of lung metastasis in animals receiving a combinatorial therapy of dabrafenib plus anti-Nodal mAb.

\section{DISCUSSION}

With the incidence of melanoma on the rise in the United States, and the challenges facing advanced-stage metastatic melanoma patients of drug resistance, disease recurrence, and tumor heterogeneity, important advances in clinical management have focused on targeted therapy. This approach is based on the foundation of scientific findings, indicating the predominance of specific molecular targets in certain patient populations. For example, approximately half of the melanoma population harbor the oncogenic BRAF mutation that leads to constitutively active BRAF-dependent cell signaling.
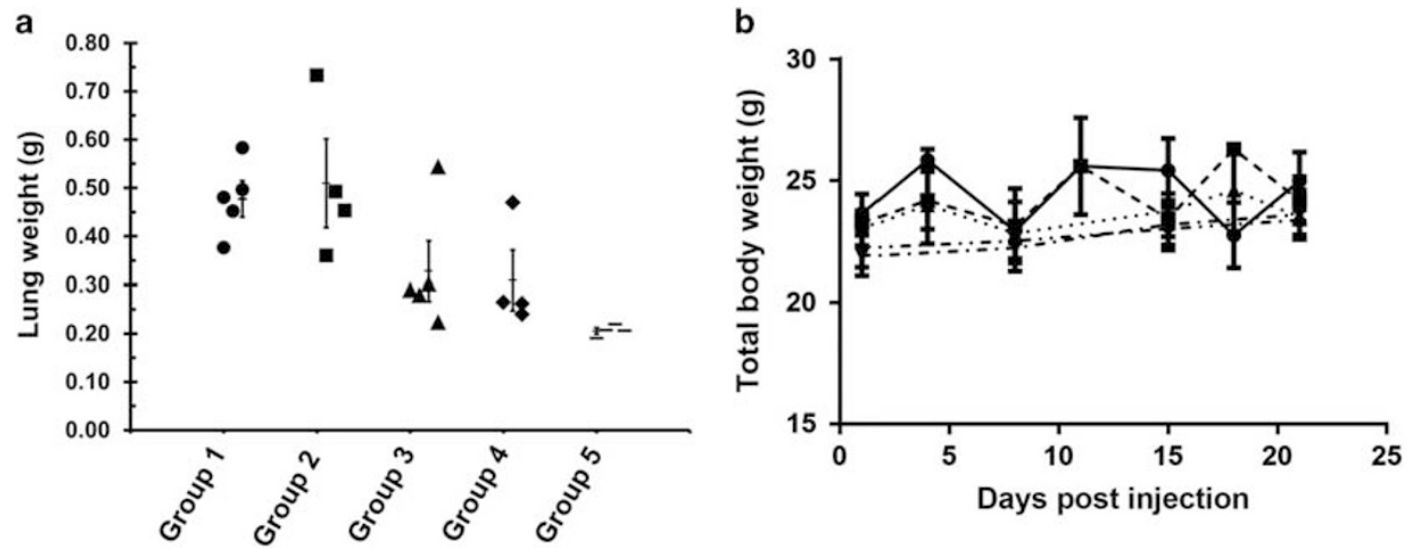

Figure 4 Combination treatment with WS65 mAb and dabrafenib significantly inhibits lung metastasis of A375SM-L1 melanoma. (a) Combination treatment with WS65 mAb plus dabrafenib (group 5) significantly reduces lung weight $(P=0.0039)$. (b) Weight loss or failure to thrive is not observed in any treatment group, indicating that all treatments are well tolerated. Data are reported as mean \pm s.e.m. in the following groups: group 1, IgG1 control

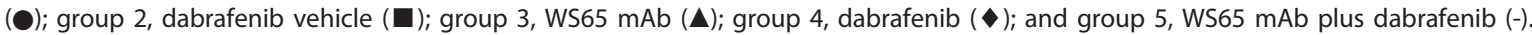

Figure 5 Morphological assessment of lungs shows significant suppression of metastasis in mice receiving combinatorial therapy. Histological analyses shows market reduction in lung tumor burden from mice treated with WS65 mAb in combination with dabrafenib (Dab+WS65) compared with the lung samples from mice in the monotherapy groups. Immunohistochemistry staining results also show almost complete loss of Nodal expression in the residual melanoma cells in the lungs from mice in the combinatorial treatment group. Background staining with isotype control lgG is shown in the inset. ( $\times 10$ original magnification). 
$H \& E$
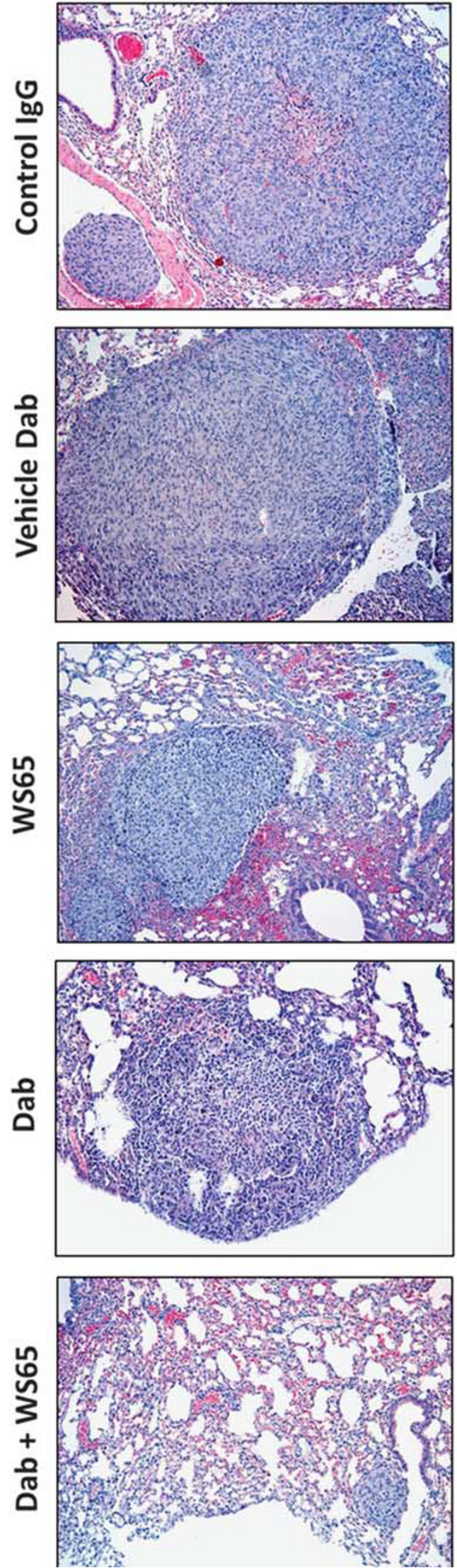

Nodal IHC
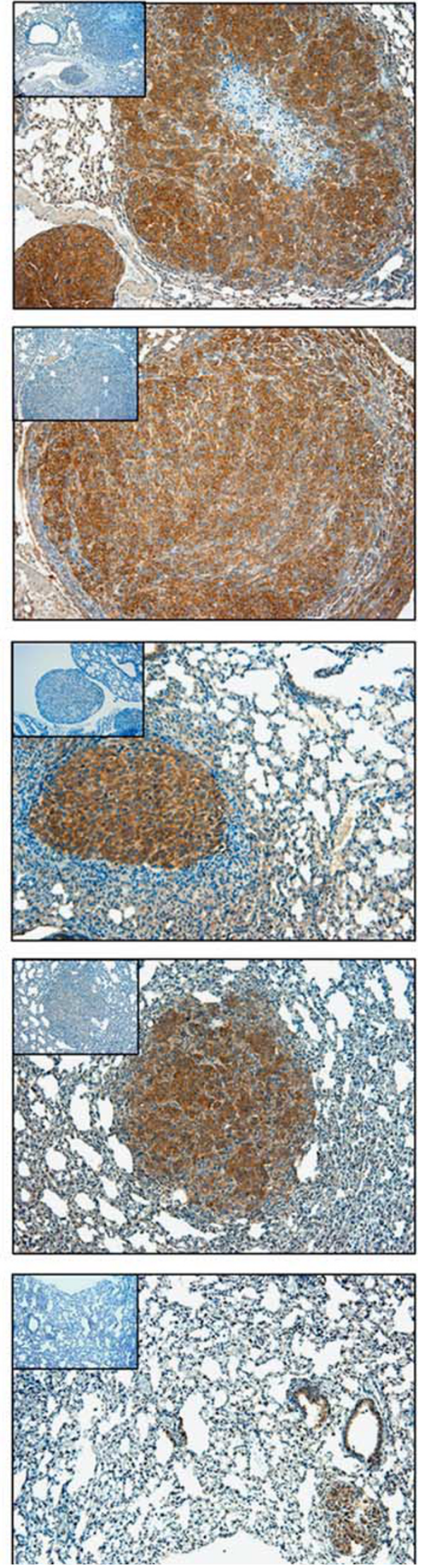
BRAFi's were developed to target these mutations; however, cutaneous toxicities have been particularly associated with BRAFi therapy. In addition, intrinsic resistance (pre-existing molecular characteristics that limit BRAFi efficacy) and acquired resistance (activation of signaling pathways that bypass BRAFi effect) remain key factors involved in BRAFi treatment failure. ${ }^{29}$

Without question, better patient selection based on the molecular profile of melanoma helps limit intrinsic resistance. Indeed, combinatorial approaches targeting putative molecular signaling molecules (MEK, PI3K-AKT-mTOR, PDGF, FGF, and EGFR) that are expressed in melanoma but unaffected during BRAFi therapy are currently being used to address acquired resistance to BRAFi. ${ }^{30}$ Most clinical trials have shown good patient response, increased progression-free survival, and in some instances increased overall survival in patients receiving combinatorial therapy. More recently, trials with BRAFi combined with immune checkpoint inhibitors are also showing promise in improving treatment outcomes compared with patients receiving BRAFi alone. ${ }^{31}$ However, adverse side effects that include autoimmunerelated toxicities with immune checkpoint inhibitors remain a major concern. Even with the best combinatorial strategy, relapse still remains an inevitable outcome in a significant number of patients due to tumor cell heterogeneity and adaptation. ${ }^{32}$

Tumor cells expressing stem cell markers and a plastic phenotype are thought to be among the most challenging to target. ${ }^{33}$ Cancer stem cells are resistant to therapy and adapt and expand over time to become major drivers of metastasis. ${ }^{34}$ One of the predominant stem cell-associated pathways that is reactivated in aggressive cancers is Nodal. ${ }^{13-16,19}$ As a member of the TGF $\beta$ superfamily, Nodal is best known for its critical role during embryogenesis, where it coordinates tissue organization, body axis specification, and maintains the pluripotency of human embryonic stem cells. ${ }^{35-37}$ Previous studies from our laboratory have shown the potential for Nodal to function as a predictive and prognostic biomarker in melanoma and breast cancer. ${ }^{16,17}$ Experimental evidence also shows that inhibition of Nodal signaling in several cancer models leads to decreases in tumorigenicity, metastasis, invasion, angiogenesis, and the plastic stem cell phenotype. ${ }^{9,17,38,39}$ Our findings also indicate that Nodal is not targeted in patients receiving the chemotherapeutic agent DTIC. ${ }^{9}$ Collectively, these observations served as a foundation for the current study, where we sought to determine whether BRAFi front-line therapy targeted Nodal. We had the advantage of using uniquely matched end-stage melanoma patient samples collected before treatment and on disease progression (that is, acquired resistance) after BRAFi therapy, which included patients diagnosed with AJCC stage IV disease where none had survived. The results indicate that similar levels of Nodal exist in matched excision biopsies PRE and POST treatment. The accompanying experimental studies using the soft agar assay and nude mouse experimental metastasis model show the most significant effects of suppressing anchorage-independent colony formation potential and metastasis, respectively, are achieved by combinatorial treatment with BRAFi plus antiNodal mAb. This novel approach of using a current front-line therapy combined with targeting a critical cancer stem cellassociated pathway, Nodal, deserves further consideration as a therapeutic option.

It is rare to have the opportunity to measure a specific marker in the same patient's tissues before and after therapy. In this study, the IHC analysis was performed in a blinded manner, where unmarked histological slides were sent to one laboratory for Nodal IHC staining, scored, and then returned to another laboratory for clinical annotation. The majority of patient samples had received dabrafenib therapy $(N=12)$, whereas one received vemurafenib, and four received a combination of dabrafenib plus trametinib (a MEK inhibitor). It is noteworthy to discover that no significant difference in Nodal IHC staining existed across the samples-regardless of treatment, patient gender, age, or type of mutation at the time of stage IV diagnosis. For the first time, these data revealed that this particular BRAFi front-line therapy does not target Nodal-expressing melanoma cells. These observations are in agreement with the previous findings from our laboratory showing that treatment of metastatic melanoma cells with either an older conventional therapy of DTIC or vemurafenib did not diminish Nodal-expressing tumor cells. ${ }^{9}$ These clinical observations allowed us to formulate and test the hypothesis that targeting Nodal together with a front-line therapy would reduce tumor growth properties and ultimately metastasis. The soft agar assay results indicated the most significant reduction in colony formation and tumorigenic growth potential using very low concentrations of dabrafenib $(1 \mathrm{nM})$ plus anti-Nodal $\mathrm{mAb}(1 \mu \mathrm{g} / \mathrm{ml})$, consistent with a more efficacious outcome with synergistic, combinatorial treatment compared with monotherapy. Based on our previous observations with DTIC treatment of melanoma cells, the residual viable cells were all Nodal positive, and sequential treatment of this population with anti-Nodal antibody resulted in adverse effects on proliferation and ultimately apoptosis. ${ }^{9}$ As Nodal-expressing tumor cells are relatively resistant to current therapies, this stem cellassociated subpopulation expands after initial front-line treatment. These mechanisms are likely involved in the current study, but were not measured. Previous preliminary observations from our laboratory indicated that treatment of the SK-MEL-28 BRAF mutated melanoma cell line with a generic BRAFi (RG7204) did not target the Nodal-expressing tumor cells, but sequential treatment with anti-Nodal $\mathrm{Ab}$ resulted in massive cell death in vitro. ${ }^{9}$ These findings were further validated and significantly expanded in the current study.

The observations generated from the experimental metastasis model using IVIS imaging, lung weight measurements, and microscopic evaluation of lungs revealed a noteworthy 
suppression of lung tumor burden in animals receiving combinatorial therapy with dabrafenib plus anti-Nodal mAb. Most notably, anti-Nodal mAb was administered only once per week i.p., whereas the dabrafenib was given three times per week via oral gavage, suggesting a light dosage regimen can yield a profound response.

Our laboratory and others have shown the significance of Nodal expression in the progression of melanoma. ${ }^{27,40}$ Most recently, we reported the effects of a novel Nodal-targeting $\mathrm{mAb}$ (3D1) in reducing growth of human melanoma xenografts in nude mice, together with diminished downstream pSmad2 signaling; ${ }^{19}$ however, this is the first in vivo study to demonstrate the efficacy of combined anti-Nodal mAb therapy with a front-line BRAFi. In fact, the WS65 antiNodal $\mathrm{mAb}$ used in the current study is different from the $3 \mathrm{D} 1 \mathrm{mAb}$ used in a previous study, thus demonstrating the universality of this $\mathrm{mAb}$ therapy. Most noteworthy, Nodal expression appears to be independent of the expression of currently targeted molecules and represents a viable alternative or complementary target in melanoma patients. We speculate that in a clinical setting, a patient would undergo therapy with front-line agents to reduce tumor mass comprised of known molecular targets, followed by antiNodal mAb treatment to suppress the residual viable Nodal-expressing tumor cells. As suggested by these data, monitoring the expression of Nodal in melanoma also could assist in stratifying the selection of patients who could potentially benefit from anti-Nodal therapy as part of a combinatorial approach with improved therapeutic outcome.

Supplementary Information accompanies the paper on the Laboratory Investigation website (http://www.laboratoryinvestigation.org)

\section{ACKNOWLEDGMENTS}

This work was supported by the H Foundation and Dixon Translational Grants Initiative (to MJCH), and NCl CCSG P30 CA060553 (to APM).

\section{DISCLOSURE/CONFLICT OF INTEREST}

$\mathrm{MJCH}$, EAS, and REBS are inventors on patents based on targeting Nodal as a cancer therapy. The remaining authors declare no conflict of interest.

1. Song $X, Z$ hao $Z$, Barber B, et al. Overall survival in patients with metastatic melanoma. Curr Med Res Opin 2015;31:987-991.

2. Gogas HJ, Kirkwood JM, Sondak VK. Chemotherapy for metastatic melanoma: time for a change. Cancer 2007;109:455-464.

3. Han SF, Liu P, Shang W, et al. The opposite-direction modulation of CD4+CD25+ Tregs and T helper 1 cells in acute coronary syndromes. Clin Immunol 2007;124:90-97.

4. Hodi FD, O'Day SJ, McDermott DF, et al. Improved survival with ipilimumab in patients with metastatic melanoma. N Engl J Med 2010;363:711-723.

5. Chapman PB, Hauschild A, Haanen JB, et al. Improved survival with vemurafenib in melanoma with BRAF V600E mutation. N Engl J Med 2011;364:2507-2516.

6. Ascierto PA, Minor D, Ribas A, et al. Phase II trial (BREAK-2) of the BRAF inhibitor dabrafenib (GSK2118436) in patients with metastatic melanoma. J Clin Oncol 2013;31:3205-3211.

7. Glaherty KT, Rober C, Hersey P, et al. Improved survival with MEK inhibition in BRAF-mutated melanoma. N Engl J Med 2012;367: 107-114.
8. Topalian SL, Hodi FS, Brahmer JR, et al. Safety, activity, and immune correlates of PD-1 antibody in cancer. N Engl J Med 2012;366: 2443-2454.

9. Hardy KM, Strizzi L, Margaryan NV, et al. Targeting Nodal in conjunction with Dacarbazine induces synergistic anticancer effects in metastatic melanoma. Mol Cancer Res 2015;13:670-680.

10. Klinac D, Gray ES, Milward $M$, et al. Advances in personalized targeted treatment of metastatic melanoma and non-invasive tumor monitoring. Front Oncol 2013;3:54.

11. Roesch A. Tumor heterogeneity and plasticity as elusive drivers for resistance to MAPK pathway inhibition in melanoma. Oncogene 2015;64:2951-2957.

12. Ascierto PA. Immunotherapies and novel combinations: the focus of advances in the treatment of melanoma. Cancer Immunol Immunother 2015;64:271-274.

13. Topczewska JM, Postovit LM, Margaryan NV, et al. Embryonic and tumorigenic pathways converge via Nodal signaling: role in melanoma aggressiveness. Nature Med 2006;12:925-932.

14. Hendrix MJC, Seftor EA, Seftor REB, et al. Reprogramming metastatic tumour cells with embryonic microenvironments. Nature Rev Cancer 2007;7:246-255.

15. Postovit LM, Margaryan NV, Seftor EA, et al. Human embryonic stem cell microenvironment suppresses the tumorigenic phenotype of aggressive cancer cells. Proc Natl Acad Sci USA 2008;105: 4329-4334.

16. Strizzi L, Postovit LM, Margaryan NV, et al. Nodal as a biomarker for melanoma progression and a new therapeutic target for clinical intervention. Expert Rev Dermatol 2009;4:67-78.

17. Strizzi L, Hardy KM, Margaryan NV, et al. Potential for the embryonic morphogen Nodal as a prognostic and predictive biomarker in breast cancer. Breast Cancer Res 2012;14:R75.

18. Bodenstine TM, Chandler GC, Seftor REB, et al. Plasticity underlines tumor progression: role of Nodal signaling. Cancer Met Rev 2016;35: 21-39.

19. Strizzi L, Sandomenico A, Margaryan NV, et al. Effects of a novel Nodaltargeting monoclonal antibody in melanoma. Oncotarget 2015;6: 34071-34086.

20. Long GV, Menzies AM, Nagrial AM, et al. Prognostic and clinicopathologic associations of oncogenic BRAF in metastatic melanoma. J Clin Oncol 2011;29:1239-1246.

21. Lyle M, Haydu LE, Mensies AM, et al. The molecular profile of metastatic melanoma in Australia. Pathology 2016;48:188-193.

22. Wilmott JS, Long GV, Howle JR, et al. Selective BRAF inhibitors induce marked T-cell infiltration into human metastatic melanoma. Clin Cancer Res 2012;18:1386-1394.

23. Long GV, Wilmott JS, Haydu LE, et al. Effects of BRAF inhibitors on human melanoma tissue before treatment, early during treatment, and on progression. Pigment Cell Melanoma Res 2013;26:499-508.

24. Gaddameedji S, Kemp MG, Reardon JT, et al. Similar nucleotide excision repair capacity in melanocytes and melanoma cells. Cancer Res 2010;70:4922-4930.

25. Matsumoto S, Tanaka F, Sato K, et al. Monitoring with a non-invasive bioluminescent in vivo imaging system of pleural metastasis of lung carcinoma. Lung Cancer 2009;66:75-79.

26. Goldman SJ, Chen E, Taylor R, et al. Use of the ODD-luciferase transgene for the non-invasive imaging of spontaneous tumors in mice. PLoS One 2011;6:e18269.

27. $\mathrm{Yu} \mathrm{L}$, Harms PW, Pouryazdanparast $\mathrm{P}$, et al. Expression of the embryonic morphogen Nodal in cutaneous melanocytic lesions. Mod Pathol 2010;23:1209-1214.

28. Mendoza A, Gharpure R, Dennis J, et al. A novel noninvasive method for evaluating experimental lung metastasis in mice. J Am Assoc Lab Anim Sci 2013;52:584-589.

29. Zhang W. BRAF inhibitors: the current and the future. Curr Opin Pharmacol 2015;23:68-73.

30. Spagnolo F, Ghiorzo P, Queirolo P. Overcoming resistance to BRAF inhibition in BRAF-mutated metastatic melanoma. Oncotarget 2014;5: 10206-10221.

31. Ashworth MT, Daud Al. Combinatorial approach to treatment of melanoma. Hematol Oncol Clin North Am 2014;28:601-612.

32. Ugurel S, Röhmel J, Ascierto PA, et al. Survival of patients with advanced metastatic melanoma: The impact of novel therapies. Eur J Cancer 2016;53:125-134. 
33. Yang M, Liu P, Huang P. Cancer stem cells, metabolism, and therapeutic significance. Tumour Biol 2016;37:5735-5742.

34. Murphy GF, Wilson BJ, Girouard SD, et al. Stem cells and targeted approaches to melanoma care. Mol Aspects Med 2014;39:33-49.

35. Arnold SJ, Robertson EJ. Making a commitment: cell lineage allocation and axis patterning in the early mouse embryo. Nat Rev Mol Cell Biol 2009;10:91-103.

36. Schier AF. Nodal morphogens. Cold Spring Harb Perspect Biol 2009;1: a003459.

37. Paulkin S, Vallier L. Activin/Nodal signaling in stem cells. Development 2015;142:607-619.
38. Quail DF, Seigers GM, Jewer $M$, et al. Nodal signaling in embryogenesis and tumourigenesis. Int J Biochem Cell Biol 2013;45: 885-898.

39. Lonardo E, Hermann PC, Mueller MT, et al. Nodal/Activin signaling drives self-renewal and tumorigenicity of pancreatic cancer stem cells and provides a target for combined drug therapy. Cell Stem Cell 2011;9:433-446.

40. Smalley KS, Haass NK, Brafford PA, et al. Multiple signaling pathways must be targeted to overcome drug resistance in cell lines derived from melanoma metastases. Mol Cancer Ther 2006;5: $1136-1144$. 